Research Article

\title{
Comparison of clinical evaluation of post-operation patients of open reduction internal fixation (ORIF) plating proximal humerus using conventional methods and minimally invasive plate osteosynthesis (MIPO) in Surabaya
}

\author{
Musa Arafah $^{1 *}$, Heri Suroto ${ }^{2}$, Erwin Ramawan ${ }^{3}$ \\ 1,2,3) Department of Orthopaedic and Traumatology, Faculty of Medicine Universitas Airlangga - Dr. Soetomo \\ General Academ ic Hospital, Surabaya, Indonesia
}

\begin{tabular}{|c|c|}
\hline \multicolumn{2}{|c|}{ A R T I C L E I N F O } \\
\hline $\begin{array}{l}\text { Submitted } \\
\text { Accepted } \\
\text { Published }\end{array}$ & $\begin{array}{l}\text { : July } 2019 \\
\text { : June } 2020 \\
\text { : July } 2020\end{array}$ \\
\hline Keywords: & \\
\hline $\begin{array}{l}\text { proximal hu } \\
\text { Invasive } \\
\text { (MIPO), Op } \\
\text { Fixation (OI }\end{array}$ & $\begin{array}{l}\text { leral fracture, Minimal } \\
\text { late Osteosynthesis } \\
\text { IF) Reduction Internal } \\
\text { IF) }\end{array}$ \\
\hline
\end{tabular}

*Correspondence:

musa_shi911@yahoo.com

\begin{abstract}
There are several kinds of approaches in the installation of implants for proximal humerus therapy. At present, minimally invasive surgery is gaining in popularity; this is supported by increasingly good technological developments to optimal the postoperative outcome is more optimal than conventional methods. This study used an analytic retrospective design with samples of postORIF Plating MIPO and posted ORIF due to proximal humeral fracture. Evaluation using the instrument of VAS Score, ASES Score, and measurement of range of motion. Statistical tests showed that there was a significant difference in the VAS score at the first evaluation $(\mathrm{p}=$ 0.002); the last review was not significant. In ASES Score, abduction, flexion, and external rotation, there were significant differences during the first and last evaluations. Adduction, extension, and internal rotation have no significance. The method of minimally invasive plate osteosynthesis (MIPO) on proximal humeral operative fracture therapy had a better clinical outcome and operating time than post-ORIF plating with conventional methods. As well, the MIPO method on proximal humeral fracture operative therapy based on radiological features has the same union rates compared to post ORIF plating with conventional methods. The method of minimally invasive plate osteosynthesis (MIPO) in operative therapy of neer 2 and 3 proximal humeral fracture has a better clinical outcome than conventional methods.
\end{abstract}




\section{INTRODUCTION}

Proximal humeral fracture is one of the most common fractures in osteoporosis patients with an incidence rate of 63 to 105 fractures per 100,000 populations each year (Bucholz et al., 2010; Solomon, Nagayam, and Warwick, 2010). There are many choices of therapeutic modalities that can be used for proximal humeral fractures depending on the severity, in general, the management of the fracture can be divided into two, namely through nonoperative and operative, $80 \%$ proximal humeral fracture is non-displaced or minimally displaced fracture so that it can be treated nonoperatively (Maier et al., 2014).

There are several kinds of approaches in implant installation for proximal humerus therapy, both with conventional to minimally invasive operating techniques. At present minimally invasive surgery is a popular technique that is supported by the development of improved imaging radiographic technology and the emergence of mono / polyaxial locking plate, the hope, of course, is that the postoperative outcome which is more optimal than the conventional method is the decrease of soft tissue trauma which leads to a decrease in the ratio of complications, reduced postoperative pain, less periarticular adhesion, and better joint function (Ruchholtz et al., 2011; Ismail et al., 2012)

Therefore, in this study we aim to compare the functional outcome method with the conventional method that has been used so far, it is hoped that this study can provide additional references, especially in handling proximal humeral fractures.

\section{METHODS}

This study used a descriptive, retrospective study design - the sample of the study that met the criteria for sample acceptance and rejection consisting of 18 samples. The sample used was posted ORIF Plating MIPO and post ORIF patients. The conventional Plating Approach was caused by a proximal humeral fracture that matched Neer's criteria (Figure 1) in RSUD Dr. Soetomo from January 2015 to September 2018 and is willing to be the subject of research and fulfill the inclusion criteria.

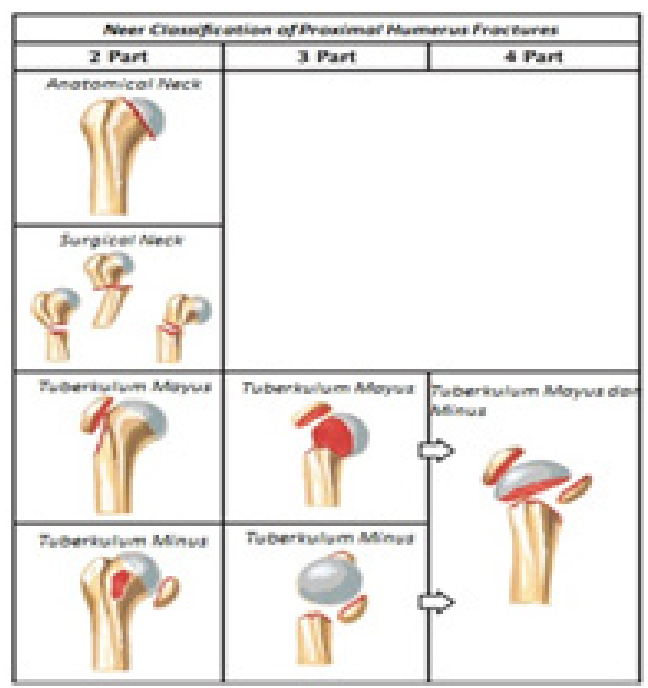

Figure 1. Neer's Classification in humerus proximal fracture (Thompson JC, 2010)

The inclusion criteria in this study posted ORIF Plating MIPO and posted ORIF patients. The conventional Plating Approach was caused by proximal humeral fractures that matched the criteria of Neer 2 and 3 with a surgical evaluation time of three months as many as two evaluation times with a range of evaluation is three months. Patients are willing to be the subjects of the study. Exclusion criteria in this study are patients with upper extremity neurological disorders and patients with multi fractures around the shoulder joint. The sampling technique was carried out by taking patient data post-ORIF Plating MIPO or post ORIF Plating conventional Approach caused by proximal humerus fractures according to Neer's criteria at Dr. General Hospital. Soetomo in the city of Surabaya. Selected patients will be compared clinical evaluations using a Visual 
Analog Scale (VAS), American Shoulder and Elbow Surgeon (ASES) Score questionnaire, and range of motion (ROM) measurements. The sample size was carried out by taking data post-ORIF Plating MIPO and post ORIF conventional Plating Approach, caused by proximal humeral fractures that matched Neer's criteria at RSUD Dr. Soetomo. All the data patients were evaluated. The nonparametric Wilcoxon and Mann-Whitney-U test were used to determine significant differences between samples. $\mathrm{P}<0.05$ was taken as an indication of significance. All statistical analyses were performed using SPSS version 22.0 software. The ethics committee approved this research of RSUD Dr. Soetomo Surabaya with ethics number 0967 / KEPK / II / 2019

\section{RESULTS}

In this study, there were 18 samples with a ratio between men and women of 1:1. The youngest patient is 30 years old, and the oldest is 78 years old, with an average sample of 50 years old. The detail of the sample characteristics was shown in Table 1.

The selected samples were operated with two treatments, namely MIPO and Conventional. In this study between conventional MIPO vs. normality test tests with various variables, among others, measuring pain (Table 2), ASES score and movement in the humeral extension, internal rotation, and external rotation, after that the abnormal distribution results in all of these variables except for the abduction variable obtained values with normal distribution, $\mathrm{P}$ $>0.05$ so to test whether there is a difference between conventional vs. MIPO on variable abduction using Independent Sample t-Test while variables with abnormal distribution using Mann Whitney. The statistical results were shown in Table 3.

Table 1. Characteristics of the sample.

\begin{tabular}{cc}
\hline \multicolumn{1}{c}{ Characteristics } & Number of Samples (percent) \\
\hline Gender & \\
Male & $9(50 \%)$ \\
Female & $9(50 \%)$ \\
& \\
Age (Mean \pm SD) & $50.50 \pm 13.61$ \\
$30-45$ & $8(44,4 \%)$ \\
$46-60$ & $5(27,8 \%)$ \\
$>60$ & $5(27,8 \%)$ \\
\hline
\end{tabular}




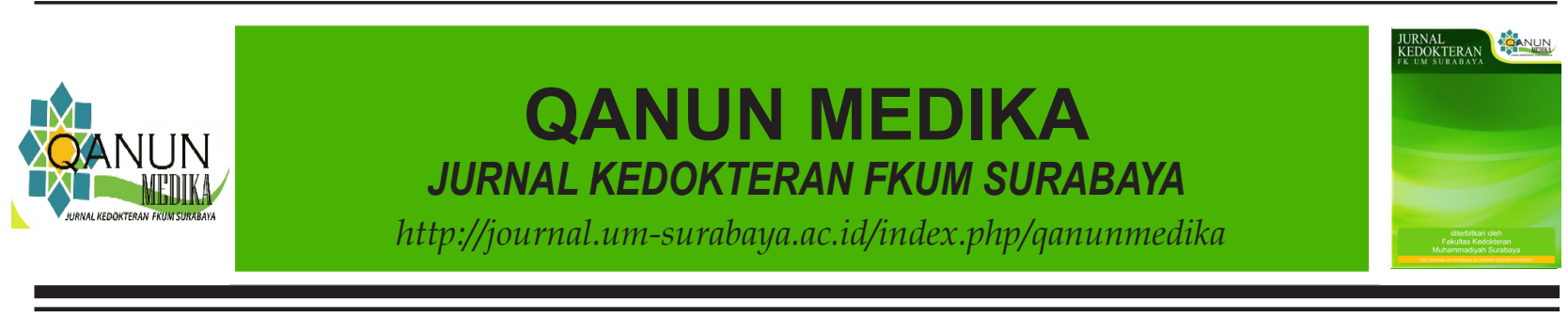

Tabel 2. VAS comparison between MIPO and conventional.

\begin{tabular}{lll}
\hline & Type of treatment & Mean \pm SD \\
\hline VAS $1^{\text {st }}$ evaluation & MIPO & $2.33 \pm 0.71$ \\
& Conventional & $3.89 \pm 0.78$ \\
VAS final evaluation & MIPO & $0.44 \pm 0.53$ \\
& Conventional & $0.44 \pm 0.53$ \\
\hline
\end{tabular}

Tabel 3. Hypotesis statistic test

\begin{tabular}{llll}
\hline Variable & & Mean \pm SD & P value (95\%CI) \\
\hline VAS Score & Initial Evaluation & $2.14 \pm 0.37$ & 0.002 \\
& final evaluation & $0.42 \pm 0.53$ & 0.916 \\
ASES Score & Initial evaluation & $92.62 \pm 1.62$ & 0.000 \\
& final evaluation & $97.70 \pm 0.66$ & 0.004 \\
Abduction & Initial evaluation & $92.85 \pm 9.51$ & 0.007 \\
\multirow{4}{*}{ Adduction } & final evaluation & $121.42 \pm 21.15$ & 0.017 \\
\multirow{3}{*}{ Extension } & Initial evaluation & $48.57 \pm 3.77$ & 0.740 \\
& final evaluation & $48.57 \pm 3.77$ & 0.740 \\
Flexion & Initial evaluation & $55.71 \pm 5.34$ & 0.638 \\
\multirow{3}{*}{ Internal rotation } & final evaluation & $58.57 \pm 3.77$ & 0.913 \\
& Initial evaluation & $94.28 \pm 5.34$ & 0.018 \\
External rotation & Initial evaluation & $54.28 \pm 7.86$ & 0.010 \\
& final evaluation & $60.00 \pm 3.77$ & 0.880 \\
& Initial evaluation & $68.57 \pm 3.77$ & 0.309 \\
& final evaluation & $68.57 \pm 3.77$ & 0.014 \\
\hline
\end{tabular}

\section{DISCUSSION}

In this study, an assessment of the VAS score was conducted between the use of MIPO and conventional. It was found that the average VAS score on the use of MIPO was 2.3 and conventional at 3.8 in the first evaluation and 0 in the use of MIPO and conventional at the last evaluation. In this study, there was a significant difference in the VAS score between the use of MIPO and conventional measures at the first evaluation $(p=0.002)$. Still, at the last evaluation, there were no differences in the VAS scores in the two groups $(p=0.916)$. This is similar to the research conducted by Liu et al., which states that there are differences in VAS scores between the use of MIPO and conventional ( $p=0.02)$ (Liu et al., 2016). No complication happened to the patient with these two types of surgery.

ASES (American Shoulder Elbow Surgeon Score) in this study was also investigated to determine the differences between the use of MIPO and conventional actions. In this study, there were significant differences in the two actions if they were associated with the ASES score. In the first evaluation, the average ASES Score of MIPO users was 89.8, and the conventional was $82.4(\mathrm{p}=0.014)$. Whereas in the last evaluation, the average ASES score of patients with MIPO users was 97, and conventional was $95(p=0.029)$. 
This is the same as research by Shang et al. $(p=0.001)$ using the ASES Score, which states that there are significant differences in ASES Score between MIPO and conventional. It used another functional outcome score method, Constant-Murley, which also showed significant functional outcome differences $(\mathrm{p}$ $=0.03$ ) (Shang LP et al., 2013). This result is different from the research conducted by Esmailiejah and Yu B et al., where there were no significant differences in functional outcomes between patients operated on using MIPO with conventional methods (Esmailiejah AA, 2015; Yu et al., 2016)

In this study, it was conducted to determine ROM function both in the ROM function of the initial action and after the action, including ROM studied, abduction, adduction, flexion, extension, internal rotation, and external rotation. In this study, between conventional vs. MIPO actions, there was a significant relationship between the initial and final evaluation ROM in ROM, namely adduction and abduction. ROM on abduction in this study with a number of angles of 110/120 in which the initial ROM evaluation was 110 degrees and finally 120 degrees with the use of MIPO was in line with the study supported by Zhou $\mathrm{ZB}$ et al., with a mean ROM of MIPO measures of 110-180 degrees and there was a match in the Vochteloo et al.'s study, et al. mentioned after the conventional operative action obtained an abduction angle of 90 degrees, wherein this study conventional measures obtained an abduction angle of 90 degrees (Vochteloo and Krekel, 2011; Zhou, Gao, and Tang, 2012).

But in this study, after being tested statistically Mann Whitney $U$ and Wilcoxon did not find a significant relationship to other ROM components both from flexion, extension, internal rotation, external rotation with two differences in working methods both with MIPO and with conventional handling both on evaluation ROM start and end.
One of the major difficulties of MIPO is obtaining adequate fracture reduction. During conventional ORIF, fracture reduction is achieved by direct visualization of the fracture and temporary stabilization with bone clamps. MIPO, on the other hand, requires indirect reduction techniques and closed fracture manipulation while plate fixation is obtained despite these challenges. Many factors can affect functional outcome between using conventional ORIF versus MIPO. Further research is needed.

\section{CONCLUSION}

Based on the discussion that has been described, the method of minimally invasive plate osteosynthesis (MIPO) on operative proximal humeral fracture therapy has a better functional outcome than post-ORIF plating with conventional methods using ASES score, because using MIPO less damage so that recovery and outcomes are faster and better.

\section{REFERENCES}

Bucholz, R. W. et al. (2010). No Title, Rockwood And Green's Fractures In Adults. 7th Edition. Philadelphia: Lippicott Williams \& Wilkins.

Esmailiejah AA, Abbasian MR, Safdari F, Ashoori K (2015). Treatment of Humeral Shaft Fractures: Minimally Invasive Plate Osteosynthesis Versus Open Reduction and Internal Fixation. Trauma Mon, 20(3).

Ismail, Boedijono, Hidayat H, Simbardjo DS. (2012). Minimal Invasive Plate Osteosynthesis ( MIPO ) Technique Using Anterolateral Approach for Treating Closed Proximal. Malaysian Orthopaedic Journal, 6(1), $18-24$.

Liu YW, Wei XE, Kuang Y, Zheng YX, Gu XF, Zhan HS, et al. (2016). Minimally Invasive Therapy \& Allied Technologies Open vs. Closed reduction combined with minimally invasive plate osteosynthesis in humeral fractures. Minimally Invasive Therapy \& Allied Technologies, 25(4), 215-221. 
Maier D, Jaeger M, Izadpanah K, Strohm PC, Suedkamp NP. (2014). Proximal Humeral Fracture Treatment in Adults. The Journal of bone and joint surgery, 96(3), 251-261.

Ruchholtz S, Hauk C, Lewan U, Franz D, Kuhne C, Zettl R. (2011). Minimally Invasive Polyaxial Locking Plate Fixation of Proximal Humeral Fractures: A Prospective Study. The Journal of Trauma Injury, Infection, and Critical Care, 71(6), 1737-1744.

Shang LP, Zhou F, Ji HQ, Zhang ZS, Liu ZS, Tian Y. (2013). Comparison of curative effects between minimally invasive locking plate internal fixation and open reduction with internal fixation for the treatment of proximal humerus fractures. Beijing Da Xue Xue Bao Yi Xue Ban., 45(5), 711-716.

Solomon, L., Nagayam, S. and Warwick, D. (2010) Apley's System Of Orthopedics and Fractures. 9th Edition. London: Hodder Arnold.
Thompson JC (2010). Netter's Concise Orthopaedic Anatomy. 2nd ed. Philadelphia: Elsevier.

Vochteloo AJH, Krekel PR. (2011). Range of motion implications of proximal humerus fractures : a case study. Eur Orthop Traumatol, 2, pp. 153-156.

Yu BF, Liu LL, Yang GJ, Zhang L, Lin Xp. (2016). Comparison of minimally invasive plate osteosynthesis and conventional plate osteosynthesis for humeral shaft fracture. Medicine (United Kingdom), 95(39), pp. 1-5.

Zhou Z, Gao Y, Tang M. (2012). Minimally invasive percutaneous osteosynthesis for proximal humeral shaft fractures with the PHILOS through the deltopectoral approach. International Orthopaedics (SICOT), 36, 23412345. 